\title{
IONIC LIQUIDS IN THE PRETREATMENT OF LIGNOCELLULOSIC BIOMASS
}

\author{
Héctor Rodríguez \\ CRETUS Institute, Department of Chemical Engineering, Universidade de Santiago de Compostela \\ E-15782, Santiago de Compostela, Spain; e-mail: hector.rodriguez@usc.es \\ (D) https://orcid.org/0000-0002-6447-3590
}

Article history: Received 2 January 2021, Received in revised form 5 February 2021, Accepted 7 February 2021, Available online 8 February 2021

\begin{abstract}
The pretreatment is a key step in the processing of lignocellulosic biomass for its transformation into chemicals and materials of biorenewable origin. Ionic liquids, with their characteristic set of unique properties, have the potential to be the basis of novel pretreatment processes with higher effectiveness and improved sustainability as compared to the current state-of-the-art processes. In this opinion paper, the author provides a perspective on possible processing strategies for this pretreatment with ionic liquids, identifying different advantages as well as challenges to be overcome.
\end{abstract}

\section{Keywords}

Ionic liquid; biomass; lignocellulose; biorefinery; pretreatment; fractionation

\section{Introduction: sustainability, lignocelluloses, biorefinery and pretreatment}

More than thirty years after the formal coinage of the term "sustainable development" [1], a tendency in the industry to shift to more sustainable processes is consolidated in the frame of the continuous improvement wheel. In 2015, the United Nations established a 2030 Agenda for Sustainable Development, including a set of sustainable development goals [2]. Although some of those goals are more specifically related to economic growth and industrial development, a strong interconnection exists among them, and their satisfactory achievement will be conditioned, for instance, by the further development of industrial processes leading to lower pollution and lower environmental impact. At present, however, a very relevant portion of plants and processes in the chemical industry and energy sector are still based on the utilization of fossil fuels or derived substances as feedstocks, with the subsequent generation of significant environmental issues and the nonsustainable depletion of natural resources. In the new context of sustainability and circular economy that impregnates the above-mentioned Agenda, there is a need for a decided switch to a new industrial platform to produce chemicals (including fuels) and materials, based on the utilization of renewable sources. The high volume of industrial production, which is expected to keep growing due to the gradual improvement of the life quality standards of our societies, poses a significant challenge on identifying the raw materials to feed such platform in a sustained fashion. An appealing candidate is lignocellulosic biomass, which is produced biorenewably by Nature at a sufficient pace as to cover the human demand of industrial production [3]. In addition, it exhibits other advantageous attributes: it presents a more homogeneous geodistribution than the fossil resources that sustain most of the global productive scheme nowadays; and, in contrast to other biorenewable sources, it avoids competition or direct interaction with the food market [4].

Although humankind has used lignocellulosic biomass in a variety of forms since prehistorical times, it was not until the identification of cellulose in the $19^{\text {th }}$ century that its intrinsic chemical richness started to be unveiled. The three major components of lignocelluloses are the three biopolymers that mainly compose the complex nonuniform 3D structure of their cell walls: cellulose, hemicellulose, and lignin. Cellulose is a linear polysaccharide consisting solely of glucose monomers, with a strong network of intra- and intermolecular hydrogen bonds; hemicellulose is an amorphous, branched polymer involving C5 (xylose, arabinose) and C6 (glucose, mannose, galactose) sugar monomers (all of which can be highly substituted by acetyl groups); and lignin is a highly branched polymer biosynthesized from up to three aromatic monomers (coniferyl, sinapyl, and $p$-coumaryl alcohols) $[3,5,6]$. While the relative composition of cellulose, hemicellulose, and lignin in the cell wall of a plant is a function of the species, tissue, and maturity, lignocellulosic biomass can be generally claimed to contain 25-50 \% cellulose, 15-35\% hemicellulose, and 10-35\% lignin [6]. The remaining fraction is made up of proteins, oils, and other organic and inorganic compounds, traditionally denoted as extractives and ashes. With all this

https://doi.org/10.32933/Actalnnovations.38.3•ISSN 2300-5599 • (C) 2021 RIC Pro-Akademia - CC BY 
chemical richness and versatility, it is clear that lignocellulosic biomass has great potential as the feedstock of reference for a biorenewable chemical platform for the industrial production of a wide portfolio of chemicals and materials. This is the basis of the concept of biorefinery [7]. However, the industrial exploitation of lignocellulosic biomass as a resource in the chemical sector has focused historically on just one of the biopolymers, namely cellulose; thus, neglecting the potential of hemicellulose and lignin to contribute to the biorefinery paradigm. It is widely accepted at present that the development of viable biorefinery schemes should consider an integral valorization of the three biopolymers $[4,8,9]$. Thus, alternative technologies are needed for a better valorization not just of cellulose but also of hemicellulose and lignin.

The efficient exploitation of lignocellulosic biomass is typically hampered by its recalcitrant character, which can be seen as the result of a natural evolution to resist degradation. This recalcitrance makes difficult the access or the disengagement of the constituent biopolymers for their suitable transformation in subsequent process stages. The recalcitrance of lignocelluloses stems mainly from the crystallinity of cellulose, the hydrophobicity of lignin, and the encapsulation of cellulose by a lignin-hemicellulose matrix [10]. To benefit from all the chemistry naturally embedded in its constituent biopolymers, the accessibility to these compounds within the lignocellulosic matrix must be improved. To assist in this accessibility improvement, the lignocellulosic feedstock is typically subjected to a pretreatment step. This is often a key step that pursues modification of the lignocellulosic structure and alteration of the pore size $[11,12]$. A variety of pretreatment methods have been developed for the processing of lignocellulosic biomass [6,13,14]: mechanical comminution, dilute acid hydrolysis, alkaline hydrolysis, steam explosion, ammonia fiber explosion, organosolv pretreatment, biological pretreatment... Despite this variety, the developed pretreatment methods present a number of drawbacks and are still far from leading to a satisfactory performance, with poorly sustainable conditions being typically involved (resulting in operational difficulties and important pollution emissions [15], as well as undesired degradation of the biopolymers), and accounting for a relevant cost within the overall processing chain [14]. There is therefore a clear need for the development of better pretreatment methods.

\section{Ionic liquids - Can they help?}

In the development of a sustainable biorefinery, the chemical products used or generated should be as environmentally friendly as possible, avoiding harmful substances. Additionally, the processes to be developed within this paradigm should maximize the transformation of the renewable raw materials into the desired final products [16,17]. In this line, with their unique characteristics and a versatile capacity for the dissolution of lignocelluloses, ionic liquids have the potential to offer attractive alternatives as auxiliary substances within the lignocellulosic biorefinery endeavor, as commented below. lonic liquids are salts with a relatively low melting or glass transition temperature (usually a mark of $100{ }^{\circ} \mathrm{C}$ is considered) [18]. Due to their ionic nature, ionic liquids have a negligible vapor pressure under usual operation conditions of most industrial processes. Thus, as opposed to conventional molecular solvents, their use would generally avoid solvent losses by evaporation (with the subsequent atmospheric pollution) and the generation of flammable atmospheres in the process plant. Although it is difficult to generalize other properties for the entire family of salts that meet the ionic liquid definition, many of them also exhibit a wide liquid range, with the upper limit determined by an acceptably good thermal stability, and a great solvation ability for a broad range of compounds. These unique characteristics have led to a prolific academic research on ionic liquids since the late 1990s, and to a great interest from the industry, resulting so far in a number of applications in diverse fields that have already reached pilot plant or full commercialization status [19].

One of the areas in which ionic liquids are showing great promise is in the processing of lignocellulosic biomass. The seminal work in this area, dating from 2002, reported the discovery of the capacity of some ionic liquids to dissolve cellulose without derivatization, in high concentrations and at relatively mild conditions [20]. In the following years, reports also appeared on the dissolution of lignin and partial or total dissolution of diverse lignocellulosic materials, including woody biomass, in ionic liquids under similarly mild conditions. Since then, the number of researchers attracted by the intersection of lignocelluloses and ionic liquids has kept growing, as well as the knowledge of the science behind the observed dissolution capacities. Broadly, ionic liquids that can dissolve cellulose are also capable of dissolving lignocelluloses; whereas there are other ionic liquids that are able to dissolve lignin but not cellulose [21-23]. Hemicellulose is, in general, more easily dissolved in conventional solvents than either cellulose or lignin, and thus no emphasis has been put on finding ionic liquids that can selectively dissolve hemicellulose [23]. 
This versatility of ionic liquids in dissolving numerous lignocellulosic materials, as well as their individual constitutive biopolymers in different extents, enables the envisioning of diverse strategies of pretreatment for the fractionation of these biopolymers and their subsequent exploitation within biorefinery schemes, as discussed in the next sections. Thanks to the mildness of the conditions and some of the inherent characteristics of ionic liquids, these novel approaches have great potential to lead to advantageous performances with respect to conventional methods.

\section{Dissolution-based strategies for the fractionation of lignocellulosic biomass with ionic liquids}

Some ionic liquids have proven their capacity to dissolve completely lignocellulosic materials, including woody biomass [5,24], under certain conditions. In such capacity, the chemical structures of both the cation and the anion constituting the ionic liquid are relevant. Whereas there is a good consensus about the need of the anion to have a high hydrogen bond basicity [5,6] (anions such as acetate, chloride, hydrogensulfate...), the required structural features for the cation remain more unclear (even though it is evident that, for a given anion, some of them work and some do not). Additionally, operating parameters such as temperature, biomass nature, particle size, solute-to-solvent load, etc. have a strong influence in this dissolution capacity [6,25]. In any case, these lignocellulose-dissolving ionic liquids enable an approach consisting of the integral dissolution of the biomass, followed by stages for the fractionation of the dissolved biopolymeric fractions, looking for the (total or partial) separation of the constitutive biopolymers. This approach is illustrated in Fig. 1. Due to the non-volatile character of both the ionic liquids and the solutes dissolved, the regeneration of the lignocellulosic fractions from the ionic liquid is not viable through unit operations based on vaporization of compounds. Liquid-liquid extraction with an organic solvent could be in principle an option, but the difficulty of finding such extraction solvent with greater preference for the dissolved fractions than the biomass-dissolving ionic liquid discourages this option. A more plausible strategy is the regeneration of the biopolymeric fractions by addition of (molecular) solvents miscible with the ionic liquid and acting as antisolvents for the dissolved material, thus precipitating it out of the solution $[5,25,26]$. The design of an adequate precipitation scheme with the appropriate set of antisolvents will lead to a total or partial fractionation of the biopolymers $[24,25,27]$, depending on the ability of the ionic liquid to actually disengage the different biopolymers in the lignocellulosic matrix upon dissolution. In Fig. 1, one such generic precipitation scheme is presented. After removal of the biopolymeric precipitates via a unit operation for solid-liquid separation (e.g., filtration), the antisolvent has to be separated from its mixture with the ionic liquid, for recycling of all components in the process. For this separation, operation units based on the creation of a vapor phase (e.g., flash distillation or evaporation) will be normally considered. Consequently, antisolvents of intermediate volatility will be desirable, thus balancing two aspects: the energy required for their removal by vaporization from the mixture with the non-volatile ionic liquid for recycling of these substances to the process; and the safety and environmental risks associated with a too volatile compound. Additionally, the proposed antisolvent should exhibit acceptably good green credentials so that the general sustainable character of the proposed process would not be compromised.

While the structures of lignin and hemicellulose remain essentially unaltered after treatment with the approach described in Fig. 1 [28], the crystallinity of cellulose in the regenerated lignocellulosic biomass fractions is lower than in the untreated material. The crystalline structure of cellulose changes from the recalcitrant native structure 'Cellulose I' to the structure 'Cellulose II' with improved processability, and there is a loss in fibrillar ordering that results in a higher amorphous component [5]. This is an aspect of great interest for a better utilization of the obtained cellulose as a precursor of e.g., derived polymers or biofuels, since the amorphous cellulose will be more accessible to chemical and enzymatic methods for its transformation.

A second approach, as an alternative to the solubilization of the entire lignocellulosic material, is based on the chemical disruption of the lignocellulose 'composite' without achieving total dissolution in the ionic liquid [5]. For example, a selective (partial) extraction of lignin from the lignocellulosic matrix can be performed with some ionic liquids able to dissolve lignin but not cellulose [5,23]. An integral scheme based on this approach is shown in Fig. 2. Since cellulose will not undergo actual dissolution, its recalcitrant crystalline character will be essentially preserved (as in most common pretreatment methods to date), as opposed to what has been commented in the paragraph above. Therefore, this approach may be more attractive in those cases in which preservation of the crystallinity is of interest and cellulose constitutes one of the final products of the biorefinery. Additionally, the set of ionic liquids that can be used in this approach is less restricted than the set of ionic liquids with the capacity 
to integrally dissolve lignocellulosic materials [21], providing a greater degree of flexibility in the ionic liquid selection. For example, ionic liquids comprising sulfonate- or sulfate-based anions may be considered (although care must be taken with ionic liquids generating less basic or even acidic environments, as a greater conversion of hemicellulose to humins and other degradation products may occur) [5]. Nevertheless, the use of molecular solvents is also necessary for the regeneration of the dissolved biopolymer fractions from the ionic liquid solution in this alternative approach.

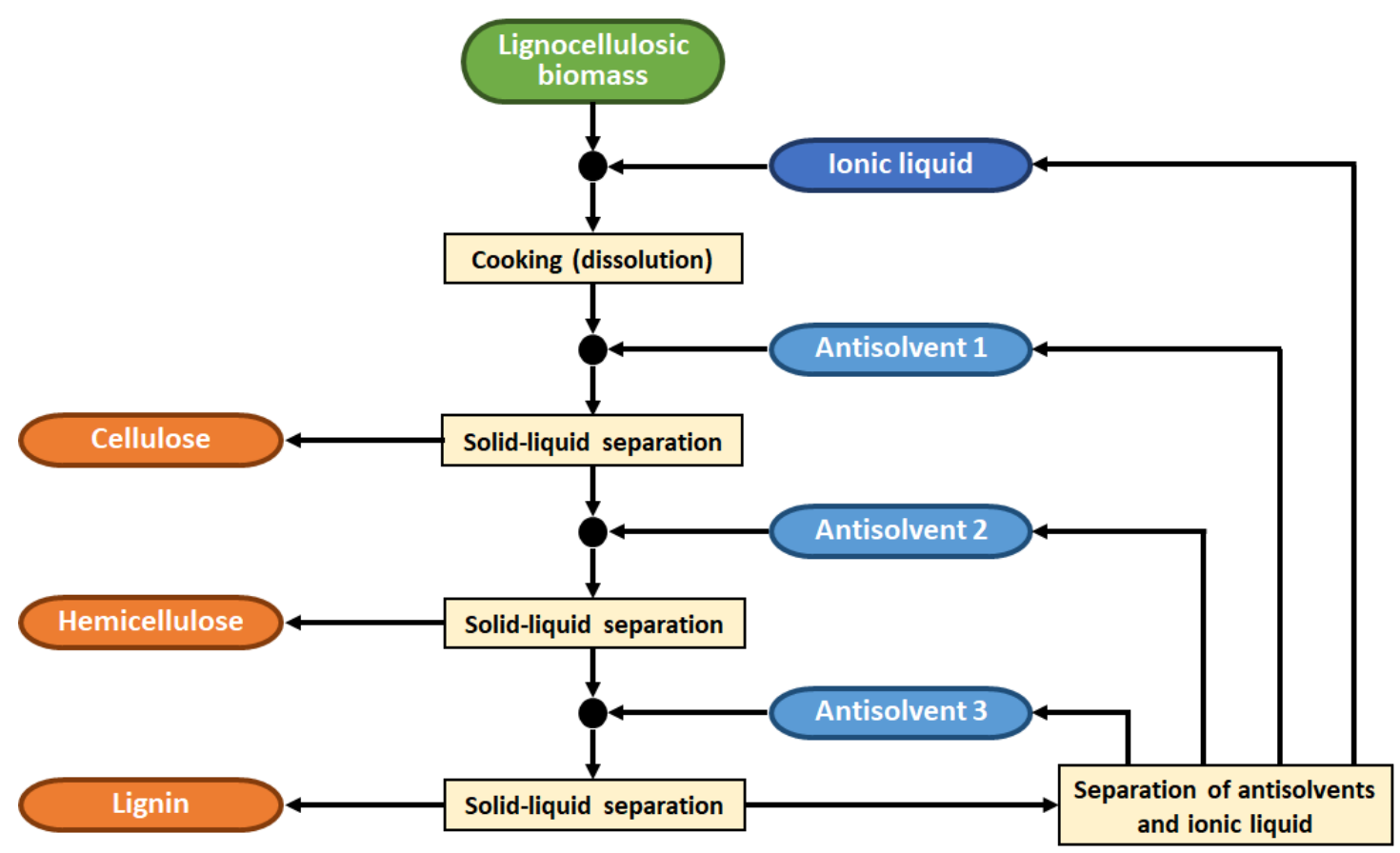

Fig. 1. Idealized scheme of the integral dissolution approach for the fractionation of lignocellulosic biomass, considering one of the many possible strategies for selective precipitation of the dissolved biopolymer fractions by addition of antisolvents.

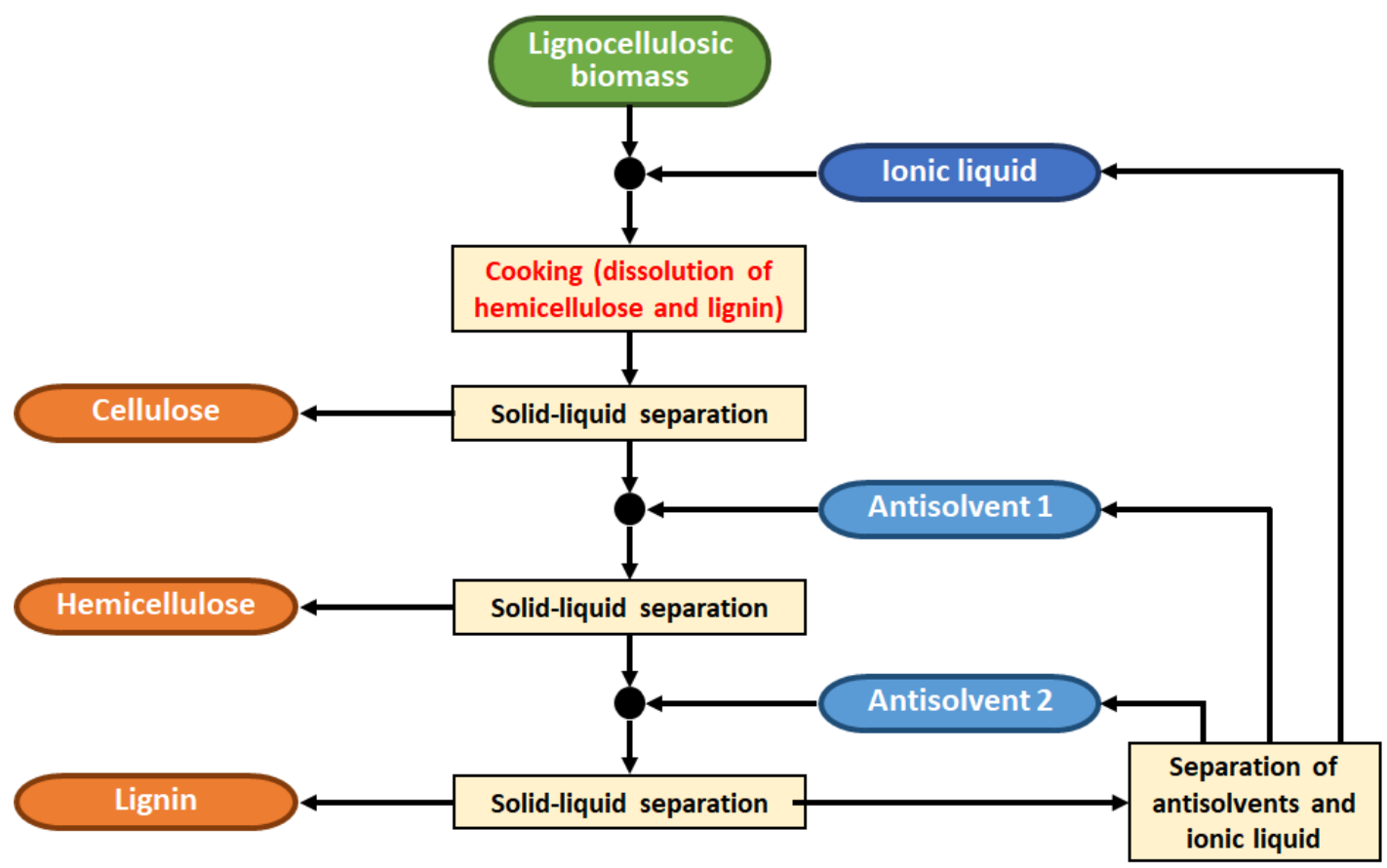

Fig. 2. Idealized scheme of a strategy based on the partial dissolution approach for the fractionation of lignocellulosic biomass. In this case, simultaneous extraction of hemicellulose and lignin from the lignocellulosic matrix is considered, although alternative strategies are possible. 
Besides the use of neat ionic liquids for the total or partial dissolution of the lignocellulosic materials, in either of the approaches the utilization of solvent systems combining ionic liquids with molecular solvents (e.g. water, acetone, aprotic polar solvents such as dimethylsulfoxide, etc.) has also been tested for a variety of biorefinery targets $[22,23]$. These combinations, except in the case of water, can be considered to lie within what is termed 'organic electrolyte solutions'. These solutions may offer some advantages, such as the lowering of the viscosity of the dissolution medium (which is one of the major drawbacks when using pure ionic liquids - see next section), or the possibility of utilizing ionic liquids or organic salts with a too high melting temperature as to be used in neat. On the other hand, the presence of the molecular cosolvent will imply the complications already commented for the antisolvents. Moreover, it must be considered that the special dissolution capacity of ionic liquids will be a sensitive function of the concentration, and the equilibrium between this dissolution capacity and a suitable set of fluid attributes for the organic electrolyte solution will be delicate.

\section{Process-related problems and some potential ways of overcoming them}

For ionic liquids to actually contribute to the fulfilment of the biorefinery paradigm through any of the process variants investigated, there are still important challenges to be addressed $[5,14,22,29]$. A first group refers to the nature of the ionic liquids themselves and will be discussed in a separate section. The second group includes challenges connected with different aspects of the processing. For example, the efficient recycling of the ionic liquid has been identified, by means of technoeconomic analyses, as one of the most critical aspects [30-32], even with projected ionic liquid costs corresponding to their scaled-up production. One of the necessary goals to succeed in this regard is the effective removal of any remaining biomass fractions from the ionic liquid after the antisolvents addition, to avoid the build-up of unwanted pretreatment byproducts that can decrease performance $[14,33]$. The cellulose-rich fractions will probably not pose a major problem, as they will be precipitated out of the solution in a reasonably easy manner; at least if an adequate engineering approach is adopted (for example, addition of the antisolvent under gentle stirring conditions) so that potential problems of gelation are avoided. However, the precipitation of hemicellulose and lignin may be more complicated, as these biopolymers have a greater tendency to non-negligible solubilities in the mixtures of ionic liquids and antisolvents. Although the fate of hemicellulose in the precipitation step of ionic liquid based pretreatments has received less attention than that of cellulose and lignin in the literature to date, it is clear at least for lignin that total precipitation (including small polymeric fragments) is likely to require excessively large amounts of antisolvent [34,35]. Subsequently, the recycling of the ionic liquid by vaporization of those amounts of antisolvent would require a prohibiting energy input. Two possible alternatives to overcome this issue are schematized in Fig. 3. A first alternative would be to remove those hard-to-precipitate fragments of biopolymer by liquid-liquid extraction with a suitable solvent (immiscible with the ionic liquid) [36]. This strategy will only make sense if a solvent is found with the capacity for effectively extracting the biopolymeric fractions from the ionic liquid medium at low solvent-to-ionic liquid ratios, as the regeneration of those fractions would be eventually done by vaporization of the extracting solvent. A second alternative may consist of the in-situ transformation (depolymerization) of the biopolymer fractions in the ionic liquid medium [34], and then recovering the generated (volatile?) products either by solvent extraction or by a vaporization technique (the latter is illustrated in Fig. 3).

The scheme proposed in Figure $3 b$ represents a step towards process intensification. Depending on the profile of the final products that constitute the target of the specific biorefinery effort, process intensification can be pushed well further, resulting in the reduction or avoidance of antisolvents and energy involved in the precipitation scheme of the biopolymer fractions from the initial pretreatment step. An example of process intensification is the one-pot integration of the stages of pretreatment, saccharification and fermentation for the production of biofuels [31,32]. In this case, the use of ionic liquids showing compatibility with the biocatalyst(s) used for the saccharification and fermentation will be a requirement.

Besides avoiding the build-up of biomass-derived substances in the ionic liquid, another key aspect for its efficient recycling (and for the economy of the entire process) is the energy required for its separation from the antisolvents after recovery of the biopolymer fractions. Obviously, this will be a function of the amount of antisolvent needed to cause the precipitation of the solutes. However, little emphasis has been put in the literature on the quantification of the minimum antisolvent to be added [26], despite its critical role in the conception and design of a process to be scaled up for application at an industrial level. As the proposed pretreatments progress towards commercialization, a focus on this quantification is gaining relevance $[34,35]$. 
The nature of the antisolvents is also relevant: in addition to the adequate balance in volatility that was already commented in the previous section, they should preferably have low/moderate specific heat and heat of vaporization, thus reducing the required energy input during the vaporization stage for the recycling. The latter is probably the Achilles heel of the use of water as antisolvent. To date, water has been the most common choice for this role in the literature, and indeed its 'intrinsic' green credentials are unbeatable. However, its high specific and vaporization heats, and a relatively high boiling temperature, imply a presumably excessive energy penalty for its elimination by vaporization at the stage of recovering the ionic liquid.
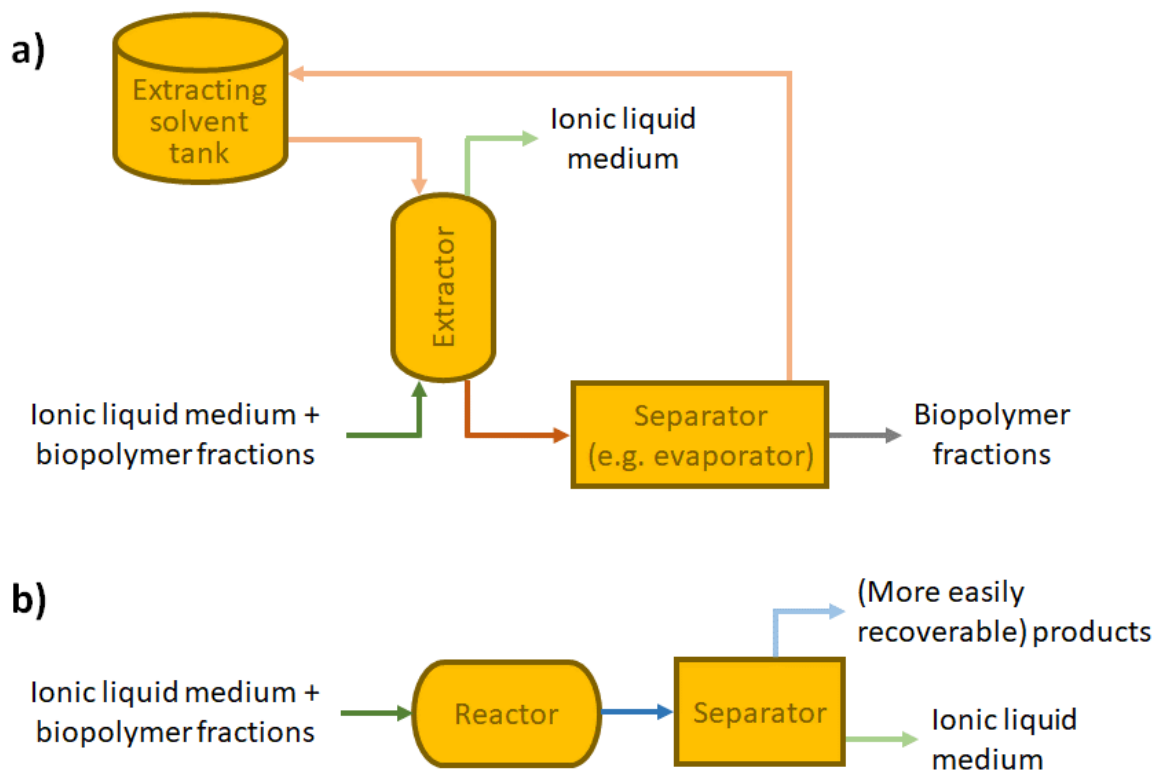

Fig. 3. Alternatives to the antisolvent strategy for the recovery of hard-to-precipitate biopolymer fractions from the ionic liquid medium: a) liquid-liquid extraction with an extracting solvent; b) in-situ transformation of the biopolymer(s) in the ionic liquid medium, with subsequent removal of the generated products.

Alternatives to the use of water or conventional organic solvents in their role as antisolvents may be of interest. The extraction with supercritical fluids, particularly $\mathrm{CO}_{2}$, was considered in general for the recovery of nonvolatile solutes from ionic liquid solutions [37], and it could be potentially interesting for the extraction of the soluble biopolymeric fractions resulting from the pretreatment of lignocellulose feedstocks. However, this technology implies high investment and operation costs, and it may render the biorefinery effort non-viable from an economic perspective. A second possibility using $\mathrm{CO}_{2}$ as antisolvent, at lower pressures, can be of application in the case of ionic liquids that react with this gas to yield a solid, as it is the case for example of the archetypal biomass-dissolving ionic liquid 1-ethyl-3-methylimidazolium acetate [38]. The economically viable reconstitution of the neat ionic liquid is still in question, and more work is required for further development of this approach.

Viscosity is another issue that can impose important limitations in processes based on ionic liquids for the pretreatment of lignocellulosic materials. Although ionic liquids often exhibit an appealing set of properties for their use as solvents in potentially sustainable processes [18,39], one of their most recurrent drawbacks is their relatively high viscosity, as compared to conventional molecular solvents. This viscosity is additionally increased upon the dissolution of (ligno)cellulosic solutes, generating relevant kinetic restrictions in the process [40]. The use of a molecular solvent as cosolvent of the ionic liquid in this kind of processes, assuming that it does not reduce significantly the dissolution capacity of the ionic liquid, would facilitate the mass transfer phenomena in the process as a result of the diminution of the viscosity [39]. Such viscosity decrease of the fluid medium would also be beneficial for the process from an engineering perspective. Moreover, the use of a cosolvent together with the ionic liquid may be a tool for the modulated control, by variation of the relative composition of ionic liquid and cosolvent, of the solubility capacity of the resulting solvent fluid, with potential application in the fractionated precipitation of the dissolved biopolymeric fractions [34,41]. This is illustrated in Fig. 4 , where it can be observed that the cosolvent becomes the antisolvent as its concentration in the fluid medium is increased. This strategy would yield in parallel the benefit of simplifying the process in the sense that it would reduce the number of auxiliary substances involved in the process, thanks to the adoption of one single substance as 
cosolvent and as fractionation antisolvent. An extra point in the utilization of this single cosolvent/antisolvent would be that the requirement of elimination of antisolvent from its mixture with the ionic liquid would be only partial, avoiding a very important percentage of the energy that would be actually required to regenerate the ionic liquid in a pure condition [42].

The implementation of improvements in the efforts to address the above-mentioned problems will help to make the pretreatment with ionic liquids viable at an industrial scale. Nevertheless, the core problem to achieve an appropriate valorization of the lignocellulosic feedstock in an integral manner with the proposed technology lies in the development of ionic liquid-based fluids capable of a cleaner fractionation of the constitutive biopolymers. The schemes in Figures 1-4 have assumed perfect separation of all three major biopolymers, but the current results have not reached that point. Moreover, the particle sizes that have been explored in most of the research to date are clearly smaller than the common particle sizes in current pretreatment processes with other technologies. If the ionic liquid technology is able to get a clean fractionation, maybe a smaller particle size than the one used in other technologies can be accepted. However, this has to be sufficiently balanced as to avoid an excessive cost of the reduction of the particle size of the biomass feedstock, which would render the entire process non-viable from an economic point of view.

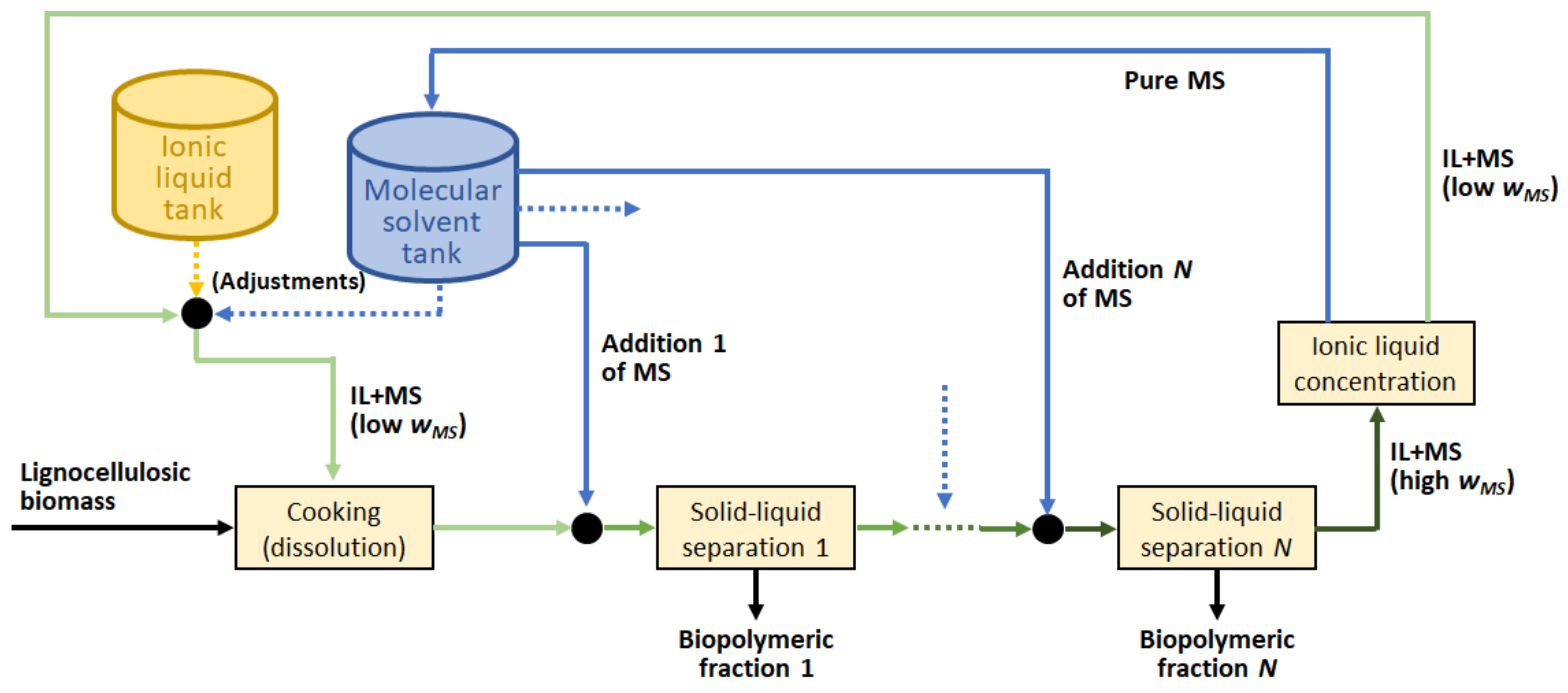

Fig. 4. Proposed use of a single substance as a cosolvent of the ionic liquid in the pretreatment step (for lowering of the viscosity) and as an antisolvent for fractionation of the dissolved biopolymeric fractions. (Legend: IL, ionic liquid; MS, molecular solvent; $w_{M S}$, mass fraction of molecular solvent in the IL+MS mixture.)

\section{Expanding the processing options: a non-dissolution approach}

The research efforts made so far on the pretreatment of lignocellulosic biomass with ionic liquids have been based mostly on the partial or total dissolution of the biomass in the ionic liquid. Due to the non-volatile nature of both the ionic liquid and the biopolymeric constituents of the biomass, this implies the use of antisolvents and problems of efficient recycling of the ionic liquid, as already commented. To avoid these problems, an alternative pretreatment scheme based on non-dissolving conditions may be envisioned (Fig. 5), depending on the objectives of the specific biorefinery context. Some ionic liquids show no capacity to dissolve (ligno)cellulosic materials but are however capable of interacting with them in the solid phase (without carrying out dissolution). The result of such interaction may be for example a reduction in crystallinity of the cellulose fraction, which will facilitate the reaction and transformation of this biopolymer in subsequent stages [43,44]. After that, the other biopolymers originally composing the lignocellulosic matrix will be likely more accessible. Importantly, due to the non-dissolving character of this approach, the use of antisolvents to precipitate dissolved fractions is not necessary in principle, and the separation of the pretreated biomass and the ionic liquid can be achieved by simple filtration. (Losses of ionic liquid impregnating the filtered solid, that will have to be washed out, can be anticipated though.)

The choice of ionic liquid for the non-dissolving pretreatment of Fig. 5 does not necessarily exclude those ionic liquids known to have the capacity to dissolve lignocellulosic materials. Such dissolution capacity may be 
hampered at certain conditions, for example at low temperature (which would be interesting from the perspective of reduction of energy consumption during the pretreatment step, while also reducing the risk of undesired thermal degradation of substances in the system). Alternatively, this dissolution capacity might be also neutralized by addition of a cosolvent, while keeping the ability of the resulting fluid system to generate a sufficient interaction with the lignocellulosic matrix as to lead to an effective pretreatment in accordance with the scheme of Fig. 5.

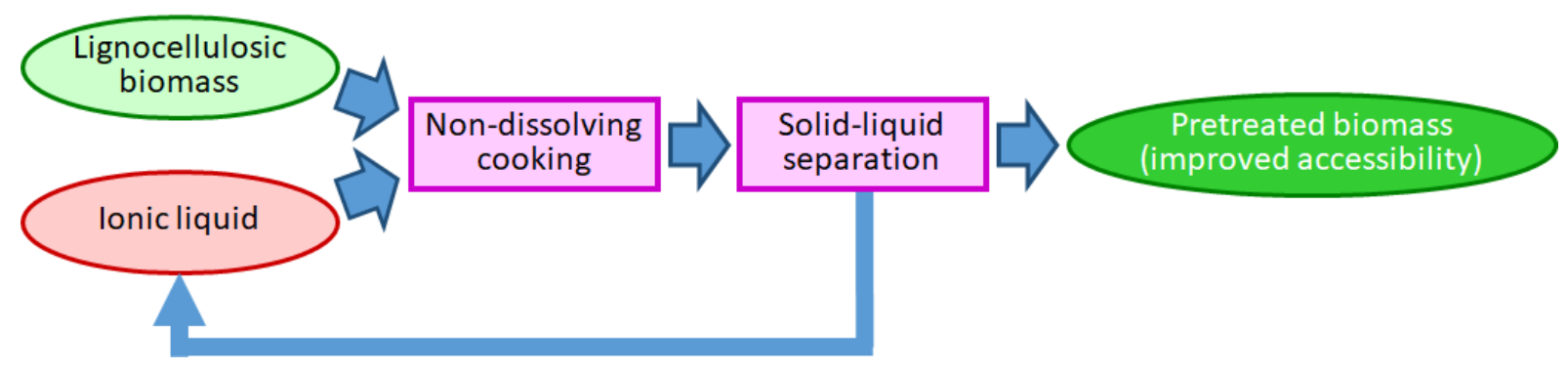

Fig. 5. Non-dissolving approach for the pretreatment of lignocellulosic biomass with ionic liquid.

\section{A focus on the general characteristics of ionic liquids themselves}

In parallel to the search of ionic liquids with better pretreatment performance in terms of dissolution, deconstruction, fractionation or related target, attention has to be paid to the intrinsic characteristics of those ionic liquids. Aspects such as cost, toxicity, biodegradability, or issues on thermal and chemical stability in the long term are matters of particular concern.

Ionic liquids are, in general, the most expensive solvents currently explored for the dissolution of lignocellulosic biomass [5,6]. Although the current commercial prices will not be representative due to the limited size of their mostly research-oriented production, the price of ionic liquids produced on a large scale will be conditioned largely by the cost of the cation and anion price [45]. With this in mind, ionic liquids with estimated costs as low as ca. $\$ 1 / \mathrm{kg}$ have been produced by protonation of cheap organic amines with standard organic or mineral acids, and applied successfully to the pretreatment of lignocelluloses [32,46]. Our experience with protic ionic liquids, nonetheless, invites to think of the existence of a non-negligible equilibrium between the ionized and neutral forms in the presence of some water (which can easily accompany the biomass up to a certain percentage). Thus, further research shedding some specific light on this aspect for those ionic liquids would be welcome, to guarantee that problems associated with strong acids and bases in some conventional pretreatment methods do not arise in here, too. Combined with the same cheap anions (e.g., chloride, acetate), some popular families of permanent cations such as tetraalkylammoniums or tetraalkylphosphoniums may lead to aprotic ionic liquids with affordable cost for industrial application as well. All in all, 'sufficiently low price' only acquires a meaning in the context of a given performance in the frame of an overall process; and it will be the corresponding economic viability study of the entire process what will dictate whether the cost of the ionic liquid is acceptable or not.

Ideally, the ionic liquids of choice should exhibit low or negligible toxicity and full biodegradability, potentially imposing a significant challenge when combining it with the list of other desired characteristics, and without compromising high performance for the intended application of pretreatment of lignocelluloses. Toxicity and biodegradability in the family of ionic liquids are tremendously diverse, and although some connection of these parameters with structural features of the ionic liquids have been identified, a full understanding remains elusive $[47,48]$. Nevertheless, it is generally acknowledged that the use of molecules from bio-renewable sources as anionic and/or cationic precursors is one of the most promising strategies to generate biocompatible ionic liquids [49]. Amino acids, non-nutritive sweeteners, glucose, carboxylic acids... could be the raw materials of choice in the ideal manufacturing of (both the cation and the anion of) bio-based ionic liquids [49]. In this line, and for the pretreatment of lignocellulosic biomass, an interesting effort has been made by developing ionic liquids with constitutive ions originating from the biomass itself [50]. Unfortunately, the associated synthetic steps involved materials and procedures with low sustainability character. The concept, though, remains certainly valid, and further research in that direction might lead to appealing results. In any case, the biocompatibility of the pretreatment fluid will be critical in those cases of process intensification where the pretreatment stage is combined with biocatalyzed stages such as saccharification or fermentation [32]. 
Chemical and thermal stability are also relevant in the selection of an ionic liquid for its utilization in a process for the pretreatment of lignocellulosic biomass. Within those ionic liquids typically investigated for such application, a paradigmatic source of undesired chemical reactivity with lignocellulosic biopolymers is represented by the acidic proton at the $\mathrm{C} 2$ position of 1,3-dialkylimidazolium cations paired with sufficiently basic anions [51,52]. A possible option to keep utilizing this kind of ionic liquids while avoiding the undesired reaction might be the pretreatment at lower temperatures, where the corresponding kinetics will not be favored. This will be obviously at the risk of rendering the biomass pretreatment less effective, or even ineffective. The suggested reduction of the processing temperature may be also motivated by a thermal degradation issue. Although the onset decomposition temperature from a dynamic run in a thermogravimetric analysis constitutes an overestimation of the real maximum temperature at which the ionic liquid can be operated, it is still taken frequently as the reference for evaluating the temperature limit of usage of the ionic liquid. For short pretreatment times, a more conservative evaluation of the onset decomposition temperature from the same dynamic thermogram (for example calculating such onset for a small decomposition percentage of the ionic liquid sample) might be fine [41]. However, and particularly for longer pretreatment stages at elevated temperature, a safer estimation of the maximum operation temperature will be obtained from isothermal thermogravimetric analyses. The latter are still barely reported in the literature, despite their capital importance in guaranteeing the thermal stability of the ionic liquid in the mid/long-term. Studies of this kind, available for some ionic liquids proposed for the pretreatment of (ligno)cellulosic materials, reveal relevant reductions in the estimated maximum operation temperature if compared to the regular onset temperature obtained by dynamic thermogravimetric analysis $[43,53]$.

One further characteristic to take into account could be the corrosive character of ionic liquids. In this regard, the scientific literature has focused mainly on the anti-corrosion properties offered by some ionic liquids, which do not correspond with those typically proposed for the pretreatment of biomass. For the latter, there is an enormous lack (and need) of information.

High viscosity can be also considered as an inherent characteristic of many ionic liquids. This issue, however, has already been discussed in a previous section.

\section{Moving forward...}

Ionic liquids offer a basis for a greatly versatile technological platform for the pretreatment of lignocellulosic biomass, in a frame of potentially improved sustainability. Promising results have been achieved over the last decade and a half. In the route towards successful industrial implementation, the search of ionic liquids with better performance must continue. In the progress to higher technology readiness levels, the following aspects should be considered:

- Improved tolerance to water in the system, as the performance of most ionic liquids explored to date decreases sharply in the presence of water.

- Satisfactory pretreatment using bigger particle sizes, so that the large energy consumption in the preparation of the feedstock for the process can be reduced [5]. A movement from sawdust to industrial chip size, or close, has to be made.

- Demonstrable flexibility to deal with the common variability in the characteristics of the supplies of a given lignocellulosic source. Ideally, this flexibility should be extended to the possibility of working with different types of lignocellulosic species (feedstock-agnostic processes).

- Fine control of the quantity of antisolvent(s) to be used, in dissolution-based approaches, for the precipitation of the (ligno)cellulosic fractions in the engineered context. This will be critical to minimize the energy penalty associated with the subsequent separation of antisolvent and ionic liquid for their recycling to the process [26].

- Choice of stable ionic liquids as biocompatible and as cheap as possible, and preferably derived from renewable raw materials [17].

The ionic liquid does not have to be necessarily at the core of the solution. The above-mentioned versatility of the ionic liquid technology enables its combination with the conventional pretreatment methods already in place [29], as well as with other alternatives under investigation (e.g. microwaves, autohydrolysis...). The use of the ionic liquid as additive in some of these scenarios, or as the main actor of just a specific step inserted in 
a general process, remains quite largely unexplored. This type of use of the ionic liquid would likely introduce a greater flexibility in terms of the cost requirements of the ionic liquid.

Most works to date on the pretreatment of lignocelluloses with ionic liquids have focused on what could be called 'simple' ionic liquids, constituted by one type of cation and one type of anion. This is the simplest and best approach to connect the observed pretreatment performances with specific ions or cation-anion pairs. Once these connections are acceptably identified, nothing prevents us from formulating a multi-ion solvent, for example by combination of 'simple' ionic liquids (or even higher-melting salts that do not qualify strictly as ionic liquids), allowing an optimization of the pretreatment performance and/or the properties of the pretreatment fluid. For instance, eutectic mixtures can be generated by such combination of 'simple' ionic liquids, integrally preserving the ionic liquid nature of the resulting fluid and enabling its utilization to pretreat biomass at a lower temperature than what would be possible for the 'parent' ionic liquids [54]. Statistical mixtures of ionic liquids are another example of multi-ion fluids, where several similar ions (e.g. dialkylimidazolium cations with alkyl substituents of different lengths) are produced in fixed molar ratios resulting from the statistical combination of the starting materials in a one-pot reactive step [55]. Most probably, the statistical mixture will perform very similarly to the 'single' ionic liquid in which it is based, with the advantage of potentially having a much lower cost of production. The existence of such one-pot synthetic procedures for the preparation, for instance, of statistical mixtures of some imidazolium-based ionic liquids from cheap starting materials may provide better opportunities for the application of these a priori too expensive ionic liquids in scaled-up processes [55].

As it was previously mentioned in this article, the combination of the ionic liquid with a molecular (co)solvent is another possibility of interest. The idea is to have a cosolvent that can strongly reduce the viscosity (thus facilitating the mass transfer processes) while not negatively affecting the pretreatment capacity in a substantial manner. It is true that, inevitably, the participation of the molecular cosolvent will imply some volatility and associated drawbacks for the pretreatment fluid. Due to the peculiarities of interaction of the ionic liquid with the lignocellulosic biomass, together with its ionic character, the best candidates among the portfolio of molecular solvents seem to be the polar aprotic solvents. Unfortunately, the solvents currently available in this category score quite badly in terms of sustainability [56], perhaps with the only exception of dimethylsulfoxide and somehow acetonitrile. Therefore, at present, it may be difficult to come up with a combination of ionic liquid and molecular solvent that be effective for the pretreatment while presenting acceptably good green credentials. Advances in the development of alternative protic apolar solvents to the classical ones, which is a general need in the chemical fields, would open new avenues for this strategy of a hybrid ionic-molecular solvent for biomass pretreatment. In any case, it must be noted that the introduction of volatile organic cosolvents or antisolvents will counteract some of the typically claimed benefits of the use of ionic liquids in processes, such as the lack of contribution to the air pollution or the non-generation of atmospheres that pose a health and safety risk in the work area. The solubility properties are clearly critical in the selection of these cosolvents/antisolvents involved in the precipitation scheme, but this selection should be also guided by a holistic consideration of their properties in the context of the process.

Other potential approaches of interest that can be pursued have already been commented in different sections of this article. One example is the possibility of having the same substance acting as a cosolvent and as antisolvent, depending on its concentration; or at least minimizing the number of working substances in the dissolution and precipitation scheme of the pretreatment process. Another example relates to process intensification, with the integration of the pretreatment stage with subsequent stages (especially if the biorefinery process is oriented towards a particular type of product, such as biofuels), through the utilization of ionic liquids with good biocompatibility.

Finally, it is worth mentioning that research on ionic liquids for the pretreatment of lignocelluloses may provide knowledge that can be beneficial for the envisioning of alternative pretreatment methods based on related solvents (e.g. imidazole [57]) or fluid mixtures (e.g. eutectic solvents resulting from mixtures involving a salt, or a hydrogen-bond donor and a hydrogen-bond acceptor $[58,59])$.

All in all, ionic liquids with their unique properties constitute a very attractive family of substances for biomass processing in a biorefinery context. However, there is a need to overcome key challenges for the development and implementation of commercially viable processes, in which the global process economy together with 
environmental and social impacts have to be properly optimized [29]. Further research efforts and intensive collaboration of industry and academia will be critical to help progressing in the scale of technology readiness levels and consolidate lignocellulosic biomass pretreatment processes based on this technology as an industrial reality of great benefit for a Society that must become necessarily more sustainable.

\section{Impact}

Ionic liquids, with their unique attributes, have the potential to be the basis of novel processes for the pretreatment of lignocellulosic biomass with improved sustainability character. The development of such pretreatment processes is expected to make the current biorefinery products more competitive, while concomitantly enabling the economic viability of other biorefinery routes for the production of new products of a biorenewable origin. Thus, the portfolio of chemicals and materials obtained from biorefinery schemes will be reinforced and expanded, improving the contribution of sustainable raw materials in the life cycle of the goods and commodities that we all use and consume as a society. This will be accompanied by the diminution of the atmospheric pollution that will result of replacing conventional organic volatile solvents with the non-volatile ionic liquids. In a similar vein, the non-generation of organic vapors by the ionic liquids will help to create safer and healthier environments for the workers in the corresponding biorefinery plants.

A better geodistribution of lignocelluloses, as compared to the current non-renewable sources, and their nonedible character, are additional characteristics that are expected to have a beneficial impact at a social level in a global context with the consolidation of the lignocellulosic biomass biorefinery paradigm.

\section{Conflict of interest}

There are no conflicts to declare.

\section{Acknowledgments}

The author is grateful to Xunta de Galicia for support through the Galician Network of Ionic Liquids (reference ED431D 2017/06) and the CRETUS Strategic Partnership (reference ED431E 2018/01), co-funded by the European Regional Development Fund.

\section{References}

[1] World Commission on Environment and Development (United Nations), Our Common Future, Oxford University Press, New York (USA), 1987.

[2] https://sustainabledevelopment.un.org/?menu=1300, (2021).

[3] G.W. Huber, S. Iborra, A. Corma, Synthesis of transportation fuels from biomass: Chemistry, catalysts, and engineering, Chem. Rev. 106 (2006) 4044-4098. https://doi.org/10.1021/cr068360d.

[4] M. FitzPatrick, P. Champagne, M.F. Cunningham, R.A. Whitney, A biorefinery processing perspective: Treatment of lignocellulosic materials for the production of value-added products, Bioresour. Technol. 101 (2010) 8915-8922. https://doi.org/10.1016/j.biortech.2010.06.125.

[5] A. Brandt, J. Gräsvik, J.P. Hallett, T. Welton, Deconstruction of lignocellulosic biomass with ionic liquids, Green Chem. 15 (2013) 550-583. https://doi.org/10.1039/c2gc36364j.

[6] Z. Usmani, M. Sharma, P. Gupta, Y. Karpichev, N. Gathergood, R. Bhat, V.K. Gupta, lonic liquid based pretreatment of lignocellulosic biomass for enhanced bioconversion, Bioresour. Technol. 304 (2020) 123003. https://doi.org/10.1016/j.biortech.2020.123003.

[7] F. Cherubini, G. Jungmeier, M. Wellisch, T. Willke, I. Skiadas, R. van Ree, E. de Jong, Toward a common classification approach for biorefinery systems, Biofuels, Bioprod. Biorefining. 3 (2009) 534-546. https://doi.org/10.1002/bbb.172.

[8] J. Zakzeski, P.C.A. Bruijnincx, A.L. Jongerius, B.M. Weckhuysen, The catalytic valorization of lignin for the production of renewable chemicals, Chem. Rev. $110 \quad$ (2010) 3552-3599. https://doi.org/10.1021/cr900354u.

[9] C.G. Yoo, X. Meng, Y. Pu, A.J. Ragauskas, The critical role of lignin in lignocellulosic biomass conversion and recent pretreatment strategies: A comprehensive review, Bioresour. Technol. 301 (2020) 122784. https://doi.org/10.1016/j.biortech.2020.122784.

[10] F.H. Isikgor, C.R. Becer, Lignocellulosic biomass: a sustainable platform for the production of bio-based chemicals and polymers, Polym. Chem. 6 (2015) 4497-4559. https://doi.org/10.1039/c5py00263j. 
[11] N. Mosier, C. Wyman, B. Dale, R. Elander, Y.Y. Lee, M. Holtzapple, M. Ladisch, Features of promising technologies for pretreatment of lignocellulosic biomass, Bioresour. Technol. 96 (2005) 673-686. https://doi.org/10.1016/j.biortech.2004.06.025.

[12] P. Kumar, D.M. Barrett, M.J. Delwiche, P. Stroeve, Methods for pretreatment of lignocellulosic biomass for efficient hydrolysis and biofuel production, Ind. Eng. Chem. Res. 48 (2009) 3713-3729. https://doi.org/10.1021/ie801542g.

[13] S. Pal, S. Padmanabhan, S. Joy, P. Kuimbhar, K.D. Trimukhe, A.J. Varma, An Overview of Studies on Pilot Scale: Lignocellulosic Biomass Pretreatment Processes Used in the Production of Second Generation Bioethanol, Trends Carbohydr. Res. 7 (2015) 41-59.

[14] B.A. Simmons, S. Singh, B.M. Holmes, H.W. Blanch, lonic liquid pretreatment, Chem. Eng. Prog. 106 (2010) 50-55.

[15] D. Klemm, B. Philipp, T. Heinze, U. Heinze, W. Wagenknecht, Comprehensive Cellulose Chemistry, WileyVCH, Weinheim, Germany, 1998. https://doi.org/10.1002/3527601937.

[16] J.H. Clark, F.E.I. Deswarte, T.J. Farmer, The integration of green chemistry into future biorefineries, Biofuels, Bioprod. Biorefining. 3 (2009) 72-90. https://doi.org/10.1002/bbb.119.

[17] R.A. Sheldon, Green and sustainable manufacture of chemicals from biomass: State of the art, Green Chem. 16 (2014) 950-963. https://doi.org/10.1039/c3gc41935e.

[18] M. Freemantle, An Introduction to lonic Liquids, The Royal Society of Chemistry, Cambridge, UK, 2010.

[19] M.B. Shiflett, (Ed.), Commercial applications of Ionic Liquids, Springer, Berlin, Germany, 2020.

[20] R.P. Swatloski, S.K. Spear, J.D. Holbrey, R.D. Rogers, Dissolution of cellose with ionic liquids, J. Am. Chem. Soc. (2002). https://doi.org/10.1021/ja025790m.

[21] K.C. Badgujar, B.M. Bhanage, Factors governing dissolution process of lignocellulosic biomass in ionic liquid: Current status, overview and challenges, Bioresour. Technol. 178 (2015) 2-18. https://doi.org/10.1016/j.biortech.2014.09.138.

[22] N. Muhammad, Z. Man, M.I.A. Mutalib, M.A. Bustam, C.D. Wilfred, A.S. Khan, Z. Ullah, G. Gonfa, A. Nasrullah, Dissolution and Separation of Wood Biopolymers Using lonic Liquids, ChemBioEng Rev. 2 (2015) 257-278. https://doi.org/10.1002/cben.201500003.

[23] Q. Hou, M. Ju, W. Li, L. Liu, Y. Chen, Q. Yang, H. Zhao, Pretreatment of lignocellulosic biomass with ionic liquids and ionic liquid-based solvent systems, Molecules. $22 \quad$ (2017) 490. https://doi.org/10.3390/molecules22030490.

[24] N. Sun, M. Rahman, Y. Qin, M.L. Maxim, H. Rodríguez, R.D. Rogers, Complete dissolution and partial delignification of wood in the ionic liquid 1-ethyl-3-methylimidazolium acetate, Green Chem. 11 (2009) 646-65. https://doi.org/10.1039/b822702k.

[25] A.M. da Costa Lopes, K.G. João, A.R.C. Morais, E. Bogel-Łukasik, R. Bogel-Łukasik, lonic liquids as a tool for lignocellulosic biomass fractionation, Sustain. Chem. Process. $1 \quad$ (2013) 3. https://doi.org/10.1186/2043-7129-1-3.

[26] H. Rodríguez, Ionic Liquids in the Context of Separation Processes, in: H. Rodríguez (Ed.), Ionic Liquid Better Seperation Processes, Springer-Verlag, Berlin, Germany, 2016: pp. 1-9. https://doi.org/10.1007/978-3-662-48520-0_1.

[27] N. Sun, H. Rodríguez, M. Rahman, R.D. Rogers, Where are ionic liquid strategies most suited in the pursuit of chemicals and energy from lignocellulosic biomass?, Chem. Commun. 47 (2011) 1405-1421. https://doi.org/10.1039/c0cc03990j.

[28] C.E. Wyman, B.E. Dale, R.T. Elander, M. Holtzapple, M.R. Ladisch, Y.Y. Lee, C. Mitchinson, J.N. Saddler, Comparative sugar recovery and fermentation data following pretreatment of poplar wood by leading technologies, Biotechnol. Prog. 25 (2009) 333-339. https://doi.org/10.1002/btpr.142.

[29] Q. Zhang, J. Hu, D.J. Lee, Pretreatment of biomass using ionic liquids: Research updates, Renew. Energy. 111 (2017) 77-84. https://doi.org/10.1016/j.renene.2017.03.093.

[30] D. Klein-Marcuschamer, B.A. Simmons, H.W. Blanch, Techno-economic analysis of a lignocellulosic ethanol biorefinery with ionic liquid pre-treatment, Biofuels, Bioprod. Biorefining. 5 (2011) 562-569. https://doi.org/10.1002/bbb.303.

[31] F. Xu, J. Sun, N.V.S.N.M. Konda, J. Shi, T. Dutta, C.D. Scown, B.A. Simmons, S. Singh, Transforming biomass conversion with ionic liquids: Process intensification and the development of a high-gravity, one-pot process for the production of cellulosic ethanol, Energy Environ. Sci. 9 (2016) 1042-1049. https://doi.org/10.1039/c5ee02940f. 
[32] J. Sun, N.V.S.N.M. Konda, R. Parthasarathi, T. Dutta, M. Valiev, F. Xu, B.A. Simmons, S. Singh, One-pot integrated biofuel production using low-cost biocompatible protic ionic liquids, Green Chem. 19 (2017) 3152-3163. https://doi.org/10.1039/c7gc01179b.

[33] P. Weerachanchai, J.M. Lee, Recyclability of an ionic liquid for biomass pretreatment, Bioresour. Technol. 169 (2014) 336-343. https://doi.org/10.1016/j.biortech.2014.06.072.

[34] M.C. Castro, H. Rodríguez, A. Arce, A. Soto, Mixtures of ethanol and the ionic liquid 1-ethyl-3methylimidazolium acetate for the fractionated solubility of biopolymers of lignocellulosic biomass, Ind. Eng. Chem. Res. 53 (2014) 11850-11861. https://doi.org/10.1021/ie501956x.

[35] C.L. Chambon, V. Fitriyanti, P. Verdía, S.M. Yang, S. Hérou, M.M. Titirici, A. Brandt-Talbot, P.S. Fennell, J.P. Hallett, Fractionation by Sequential Antisolvent Precipitation of Grass, Softwood, and Hardwood Lignins Isolated Using Low-Cost Ionic Liquids and Water, ACS Sustain. Chem. Eng. 8 (2020) 3751-3761. https://doi.org/10.1021/acssuschemeng.9b06939.

[36] Q. Xin, K. Pfeiffer, J.M. Prausnitz, D.S. Clark, H.W. Blanch, Extraction of lignins from aqueous-ionic liquid mixtures by organic solvents, Biotechnol. Bioeng. $109 \quad$ (2012) 346-352. https://doi.org/10.1002/bit.24337.

[37] L.A. Blanchard, D. Hancu, E.J. Beckman, J.F. Brennecke, Green processing using ionic liquids and CO2, Nature. 398 (1999) 28-29. https://doi.org/10.1038/19887.

[38] P.S. Barber, C.S. Griggs, G. Gurau, Z. Liu, S. Li, Z. Li, X. Lu, S. Zhang, R.D. Rogers, Coagulation of chitin and cellulose from 1-ethyl-3-methylimidazolium acetate ionic-liquid solutions using carbon dioxide, Angew. Chemie - Int. Ed. 52 (2013) 12350-12353. https://doi.org/10.1002/anie.201304604.

[39] A. Stark, K.R. Seddon, Ionic liquids, in: A. Seidel (Ed.), Kirk-Othmer Encyclopedia of Chemical Technology, 5th ed., vol. 26, Wiley, Hoboken, New Jersey, USA, 2007: pp. 836-920.

[40] J.M. Andanson, A.A.H. Pádua, M.F. Costa Gomes, Thermodynamics of cellulose dissolution in an imidazolium acetate ionic liquid, Chem. Commun. 51 (2015) 4485-4487. https://doi.org/10.1039/c4cc10249e.

[41] M.C. Castro, A. Arce, A. Soto, H. Rodríguez, Influence of Methanol on the Dissolution of Lignocellulose Biopolymers with the Ionic Liquid 1-Ethyl-3-methylimidazolium Acetate, Ind. Eng. Chem. Res. 54 (2015) 9605-9614. https://doi.org/10.1021/acs.iecr.5b02604.

[42] M.K. Wojtczuk, N. Caeiro, H. Rodríguez, E. Rodil, A. Soto, Recovery of the ionic liquids [C2mim][OAc] or [C2mim][SCN] by distillation from their binary mixtures with methanol or ethanol, Sep. Purif. Technol. 248 (2020) 117103. https://doi.org/10.1016/j.seppur.2020.117103.

[43] C.A. Pena, A. Soto, A.W.T. King, H. Rodríguez, Improved Reactivity of Cellulose via Its Crystallinity Reduction by Nondissolving Pretreatment with an Ionic Liquid, ACS Sustain. Chem. Eng. 7 (2019) 91649171. https://doi.org/10.1021/acssuschemeng.8b06357.

[44] D. Rico del Cerro, T. V. Koso, T. Kakko, A.W.T. King, I. Kilpeläinen, Crystallinity reduction and enhancement in the chemical reactivity of cellulose by non-dissolving pre-treatment with tetrabutylphosphonium acetate, Cellulose. 27 (2020) 5545-5562. https://doi.org/10.1007/s10570-020-03044-6.

[45] C. Hilgers, P. Wasserscheid, Quality aspects and other questions related to commercial ionic liquid production., in: P. Wasserscheid, T. Welton (Eds.), Ionic Liquids in Synthesis, Wiley-VCH, Weinheim, Germany, 2003: pp. 21-33.

[46] L. Chen, M. Sharifzadeh, N. Mac Dowell, T. Welton, N. Shah, J.P. Hallett, Inexpensive ionic liquids: [HSO4]-based solvent production at bulk scale, Green Chem. 16 (2014) 3098-3106. https://doi.org/10.1039/c4gc00016a.

[47] M. Petkovic, K.R. Seddon, L.P.N. Rebelo, C.S. Pereira, lonic liquids: A pathway to environmental acceptability, Chem. Soc. Rev. 40 (2011) 1383-1403. https://doi.org/10.1039/c004968a.

[48] K.S. Egorova, V.P. Ananikov, Toxicity of ionic liquids: Eco(cyto)activity as complicated, but unavoidable parameter for task-specific optimization, ChemSusChem. 7 (2014) 336-360. https://doi.org/10.1002/cssc.201300459.

[49] J.M. Gomes, S.S. Silva, R.L. Reis, Biocompatible ionic liquids: Fundamental behaviours and applications, Chem. Soc. Rev. 48 (2019) 4317-4335. https://doi.org/10.1039/c9cs00016j.

[50] A.M. Socha, R. Parthasarathi, J. Shi, S. Pattathil, D. Whyte, M. Bergeron, A. George, K. Tran, V. Stavila, S. Venkatachalam, M.G. Hahn, B.A. Simmons, S. Singh, Efficient biomass pretreatment using ionic liquids derived from lignin and hemicellulose, Proc. Natl. Acad. Sci. U. S. A. 111 (2014) E3587-E3595. https://doi.org/10.1073/pnas.1405685111. 
[51] T. Liebert, T. Heinze, Interaction of ionic liquids with polysaccharides 5 . Solvents and reaction media for the modification of cellulose, BioResources. 3 (2008) 576-601. https://doi.org/10.15376/biores.3.2.576601.

[52] G. Ebner, S. Schiehser, A. Potthast, T. Rosenau, Side reaction of cellulose with common 1-alkyl-3methylimidazolium-based ionic liquids, Tetrahedron Lett. 49 (2008) 7322-7324. https://doi.org/10.1016/j.tetlet.2008.10.052.

[53] M.T. Clough, K. Geyer, P.A. Hunt, J. Mertes, T. Welton, Thermal decomposition of carboxylate ionic liquids: Trends and mechanisms, Phys. Chem. Chem. Phys. 15 (2013) 20480-20495. https://doi.org/10.1039/c3cp53648c.

[54] O. Stolarska, A. Pawlowska-Zygarowicz, A. Soto, H. Rodríguez, M. Smiglak, Mixtures of ionic liquids as more efficient media for cellulose dissolution, Carbohydr. Polym. 178 (2017) 277-285. https://doi.org/10.1016/j.carbpol.2017.09.025.

[55] G. Gurau, H. Wang, Y. Qiao, X. Lu, S. Zhang, R.D. Rogers, Chlorine-free alternatives to the synthesis of ionic liquids for biomass processing, Pure Appl. Chem. 84 (2012) 745-754. https://doi.org/10.1351/PACCON-11-11-10.

[56] C.M. Alder, J.D. Hayler, R.K. Henderson, A.M. Redman, L. Shukla, L.E. Shuster, H.F. Sneddon, Updating and further expanding GSK's solvent sustainability guide, Green Chem. 18 (2016) 3879-3890. https://doi.org/10.1039/c6gc00611f.

[57] A. Toscan, A.R.C. Morais, S.M. Paixão, L. Alves, J. Andreaus, M. Camassola, A.J.P. Dillon, R.M. Lukasik, Effective Extraction of Lignin from Elephant Grass Using Imidazole and Its Effect on Enzymatic Saccharification to Produce Fermentable Sugars, Ind. Eng. Chem. Res. 56 (2017) 5138-5145. https://doi.org/10.1021/acs.iecr.6b04932.

[58] C.W. Zhang, S.Q. Xia, P.S. Ma, Facile pretreatment of lignocellulosic biomass using deep eutectic solvents, Bioresour. Technol. 219 (2016) 1-5. https://doi.org/10.1016/j.biortech.2016.07.026.

[59] X. Tang, M. Zuo, Z. Li, H. Liu, C. Xiong, X. Zeng, Y. Sun, L. Hu, S. Liu, T. Lei, L. Lin, Green Processing of Lignocellulosic Biomass and Its Derivatives in Deep Eutectic Solvents, ChemSusChem. 10 (2017) 26962706. https://doi.org/10.1002/cssc.201700457. 